\title{
Expression and clinical significance of IL-33 and its receptor ST2 in children with obstructive sleep apnea syndrome
}

\author{
Zibo Zhang ${ }^{1 \#}$, Liang Li ${ }^{2 \#}$, Linsheng Zhao ${ }^{3}$, Guangping Liu ${ }^{2}$, Fei Han ${ }^{4}$, Juan $\mathrm{Du}^{1}, \mathrm{Li}_{\mathrm{Liu}}{ }^{1}$ \\ ${ }^{1}$ Immunology Division of General Internal Medicine, Tianjin Children's Hospital (Tianjin University Children's Hospital), Tianjin, China; \\ ${ }^{2}$ Department of Otolaryngology, Tianjin Children's Hospital (Tianjin University Children's Hospital), Tianjin, China; ${ }^{3}$ Department of Pathology, \\ Tianjin Children's Hospital (Tianjin University Children's Hospital), Tianjin, China; ${ }^{4}$ Graduate School of Tianjin Medical University, Tianjin, China \\ Contributions: (I) Conception and design: Z Zhang, L Li; (II) Administrative support: L Liu; (III) Provision of study materials or patients: L Zhao, G \\ Liu; (IV) Collection and assembly of data: F Han; (V) Data analysis and interpretation: J Du, L Liu; (VI) Manuscript writing: All authors; (VII) Final \\ approval of manuscript: All authors. \\ "These authors contributed equally to this work. \\ Correspondence to: Li Liu. Immunology Division of General Internal Medicine, Tianjin Children's Hospital (Tianjin University Children's Hospital), \\ 225 Race Course Road, Tianjin 300074, China. Email: may13_cn@126.com.
}

Background: Obstructive sleep apnea syndrome (OSAS) is characterized by a majority population of respiratory sleep disorders, which consists of simple snoring as well as increased upper airway resistance syndrome. Adenoid hypertrophy has been suggested as the main cause of OSAS in children. The role of interleukin-33 (IL-33) and its receptor suppressor of tumorigenicity 2 (ST2) in a variety of pediatric allergic diseases has been confirmed. We hypothesized that IL-33/ST2 path way might play a pivotal role in the pathogenesis of adenoid hypertrophy-associated OSAS in children.

Methods: A total of 40 children undergoing adenoidectomy due to OSAS in the Otolaryngology of Tianjin Children's Hospital were selected as the study participants. The quantity of IL-33 and ST2 positive cells in adenoids was detected by immunohistochemical (IHC) streptavidin-peroxidase conjugate (SP) method.

Results: The IL-33 positive cells were mainly distributed in the submucosa epithelium and vascular endothelium, and expressed in the nucleus and cytoplasm. Meanwhile, ST2 positive cells were primarily observed in the mucosa and expressed in the nucleus and cytoplasm, with a little expression of intercellular substance. There was a positive correlation between the proportion of adenoids in the posterior nostril diameter and the number of IL-33 positive cells. The expression of IL-33 in adenoids was positively correlated with the level of ST2 $(\mathrm{r}=0.809, \mathrm{P}=0.000)$. The expression of IL-33 in adenoids was positively correlated with the level of eosinophil granulocyte $(\mathrm{r}=0.859, \mathrm{P}=0.000)$. Moreover, the expression of ST2 in adenoids was positively correlated with the level of eosinophil granulocyte $(r=0.814, \mathrm{P}=0.000)$. The number of IL-33 positive cells was significantly higher in the moderate hypoxemia group than that in the mild hypoxemia group $(\mathrm{P}<0.05)$. There was no significant difference in the number of ST2 positive cells between the moderate hypoxemia group and mild hypoxemia group $(\mathrm{P}>0.05)$.

Conclusions: Both IL-33 and its receptor ST2 were expressed in adenoids of OSAS children. The severity of airway obstruction caused by adenoid hypertrophy was positively correlated with the expression of IL-33.

Keywords: Children; obstructive sleep apnea syndrome (OSAS); interleukin-33 (IL-33); suppressor of tumorigenicity 2 (ST2); adenoidal hypertrophy

Submitted Nov 08, 2021. Accepted for publication Jan 11, 2022.

doi: 10.21037/tp-21-606

View this article at: https://dx.doi.org/10.21037/tp-21-606

^ ORCID: 0000-0001-8657-5339. 


\section{Introduction}

Respiratory sleep disorders (RSD) in children represent a variable obstruction of the upper airway and distinct degrees of alteration in gas exchange during the night $(1,2)$. Obstructive sleep apnea syndrome (OSAS) is characterized by a majority population of RSD, which consists of simple snoring as well as increased upper airway resistance syndrome (UARS). Based on the third edition of the International Classification of Sleep Disorders (ICSD-3), OSAS is defined as a polysomnography (PSG)-determined obstructive respiratory disturbance index (RDI) $\geq 5$ events/h along with the typical symptoms of OSAS (such as unrefreshing sleep, daytime sleepiness, fatigue or insomnia, awakening with a gasping or choking sensation, loud snoring, or noticeable apneas), or an obstructive RDI $\geq 15$ events/h (even in the absence of symptoms) (3).

The clinical presentations of a child with OSAS are quite suggestive, with the common symptoms of habitual snoring, sleep disorders, and daytime neurobehavioral problems (4). As OSAS progresses, it can damage multiple systems in children, including the nervous and cardiovascular systems (5). Meanwhile, it could also result in cognitive or neuropsychological deficits like verbal and non-verbal reasoning $(6,7)$. It was estimated that the prevalence of this disorder is about $2 \%$ in children from 2 to 8 years of age (8). In children, OSAS is a complicated disorder with a large degree of inter- and intra-tumoral heterogeneity. Accumulating evidence from epidemiologic studies has suggested a variety of risk factors, including obesity, family history of OSAS, adenoid and/or tonsil hypertrophy, allergic rhinitis, craniofacial abnormalities, and genetics (9-12). Compared with OSAS in adults, the molecular mechanisms for children are far from satisfactorily understood.

Adenoid hypertrophy has been suggested as the main cause of OSAS (13). Adenoids are present at birth, most notably at 6-7 years of age, and gradually atrophy after 10 years of age. Due to their special anatomical location, adenoids are the first sites exposed to foreign antigens. Repeated inflammatory stimulation leads to hyperplasia, fibrosis, and hypertrophy of the adenoid tissue, resulting in the corresponding clinical symptoms (14). Interleukin-33 (IL-33) is a newly discovered member of the interleukin 1 (IL-1) family. The receptor for IL-33 is also known as suppressor of tumorigenicity 2 (ST2) (15). Expression of IL-33 is mainly in the skin, intestine, lung, digestive tract, and other barrier tissue cells (16). In recent years, the roles of the IL-33/ST2 pathway in a variety of pediatric allergic diseases have been confirmed, such as allergic rhinitis, asthma, atopic dermatitis, food allergy, and so on (17). It is worth noting that a considerable population of these children also display OSAS syndromes. Our study was the first to explore the expression of IL-33/ST2 in the adenoids of OSAS patients in children. To this end, we hypothesized that IL-33 might play a pivotal role in the pathogenesis of adenoid hypertrophy-associated OSAS in children.

We present the following article in accordance with the MDAR reporting checklist (available at https:// tp.amegroups.com/article/view/10.21037/tp-21-606/rc).

\section{Methods}

\section{Clinical specimens}

A total of 40 children (25 males and 15 females, aged 2-8 years old), who had to undergo adenoidectomy and tonsillectomy due to OSAS from January 2015 to December 2016 in Otolaryngology of Tianjin Children Hospital were randomly selected. The mean of age for all participants was $4.7 \pm 1.3$ years. The inclusion criteria were as follows: (I) diagnosis was in line with the 2007 Urumqi OSAS Draft; (II) no hormone, antihistamine, and desensitisation immunotherapy had been received within 3 months; and (III) no respiratory infectious disease within 1 month. The participants were divided into $1 / 2$ group, $2 / 3$ group, $3 / 4$ group, and $4 / 5$ group according to the degree of adenoid hypertrophy, that is, the proportion of adenoid to the inner diameter of the posterior nostril. The adenoid tissue excised during surgery was preserved by paraffin embedding after conventional fixation and dehydration. The paraffin embedded sections were used for hematoxylin and eosin (HE) staining and immunohistochemical (IHC) staining. All procedures performed in this study involving human participants were in accordance with the Declaration of Helsinki (as revised in 2013). This study was approved by the Bioethics Committee at Tianjin Children's Hospital (2021-YKY-04). A written consent form for publication of data was provided by the parents of participants.

\section{HE staining}

Under the microscope $(\times 400)$, each specimen was sectioned randomly from 5 visual fields, the number of each visual field was recorded, and then the average value of 5 visual fields was calculated as an eosinophil granulocyte count of the adenoids in each child. 


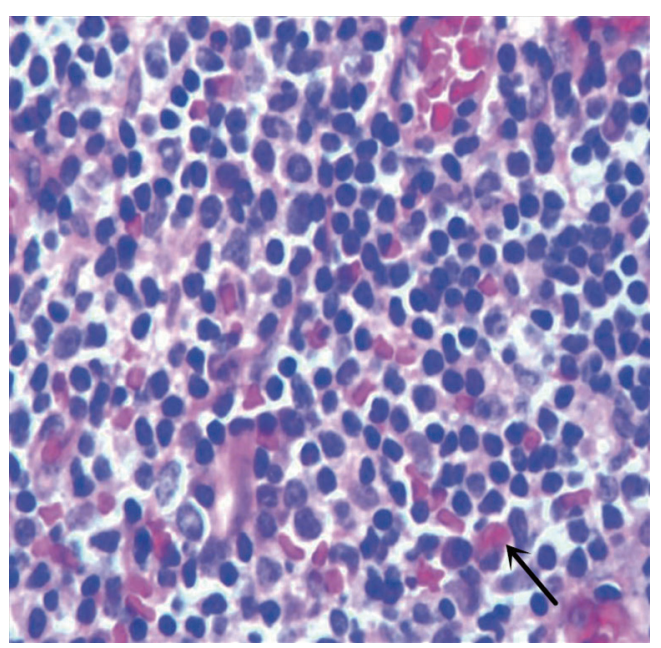

Figure $1 \mathrm{HE}$ staining of adenoids. $\times 100$. The black arrow indicates eosinophil granulocytes. HE, hematoxylin and eosin.

\section{Immunostaining}

The paraffin sections were washed with phosphate-buffered saline (PBS) solution, followed by antigen repair solution treatment and $3.0 \% \mathrm{H}_{2} \mathrm{O}_{2}$ cleaning. The specimens were then incubated at room temperature for $15 \mathrm{~min}$, washed with goat serum, and incubated at room temperature for $10 \mathrm{~min}$. The primary antibody (1:4,000 diluted antiIL-33 antibody or anti-ST2 antibody) was incubated in the refrigerator at $4{ }^{\circ} \mathrm{C}$ overnight, and the labelled $\mathrm{IgG}$ secondary antibody was taken out the next morning and incubated in the constant temperature water tank at $37^{\circ} \mathrm{C}$ for $30 \mathrm{~min}$. After rinsing with PBS solution, the 3,3'-diaminobenzidine (DAB) solution was added and the color development was observed under the microscope. Under the microscope $(\times 400)$, the reaction products labeled by color were used to localize the antigen, and the expression levels of IL-33 and ST2 were measured by positive cell count. Each slice was randomly taken from 5 visual fields, the number of positive cells was counted, and the average value was calculated.

\section{Statistical analysis}

The software SPSS 22.0 (IBM Corp., Chicago, IL, USA) and Prism 6 software (GraphPad Software Inc., La Jolla, CA, USA) were used for statistical analysis. KolmogorovSmirnov test was used to test the normality of variables. The mean and standard deviation $(\bar{x} \pm s)$ were used to express the measurement data. The $t$-test or analysis of variance was performed to compare the sample mean. The Pearson's method and Spearman's method were generated to analyze the correlation, and $\mathrm{P}<0.05$ was considered statistically significant.

\section{Results}

\section{HE staining}

There were more glands and fibers in the mucosa of the adenoids, indicating more eosinophil granulocyte infiltration. At the same time, there were less glands, fibers, and eosinophil granulocytes in the parenchyma (Figure 1).

\section{Immunostaining results}

The distribution of IL-33 positive cells in adenoids was mainly in mucosal epithelium and vascular endothelium, and IL-33 was expressed in nucleus and cytoplasm (Figure 2A). Meanwhile, the ST2 positive cells in adenoids were predominantly found in mucosa, and ST2 was mainly expressed in nucleus and cytoplasm, and a little in intercellular substance (Figure 2B).

\section{The correlation between the expression of IL-33 and ST2 in adenoids and the proportion of adenoids in the inner diameter of the posterior nostril}

According to the proportion of adenoids in the inner diameter of the posterior nostril, the adenoids were divided into $1 / 2$ group, $2 / 3$ group, $3 / 4$ group, and $4 / 5$ group. The degree of adenoid hypertrophy was graded. The correlations between the number of IL-33 and ST2 positive cells and the proportion of adenoid to the inner diameter of the posterior nostril were examined by Spearman's analysis with Prism 6 software. The results are shown in Figure 3. The Spearman coefficient between the number of IL-33 and ST2 positive cells and the proportion of adenoids in the diameter of the posterior nostril was $0.6986(\mathrm{P}<0.0001)$. These outcomes suggested that the correlation coefficient had statistical significance and the 2 variables were positively correlated. It indicates that the more severe of OSAS, the higher the expression of IL-33/ST2 in adenoids.

The expression of IL-33 was positively correlated with ST2 $(r=0.809, P=0.000)$. There was a positive correlation between the expression of IL-33 and the amount of eosinophil granulocytes in adenoids $(\mathrm{r}=0.859, \mathrm{P}=0.000)$. The expression level of ST2 was positively correlated with 

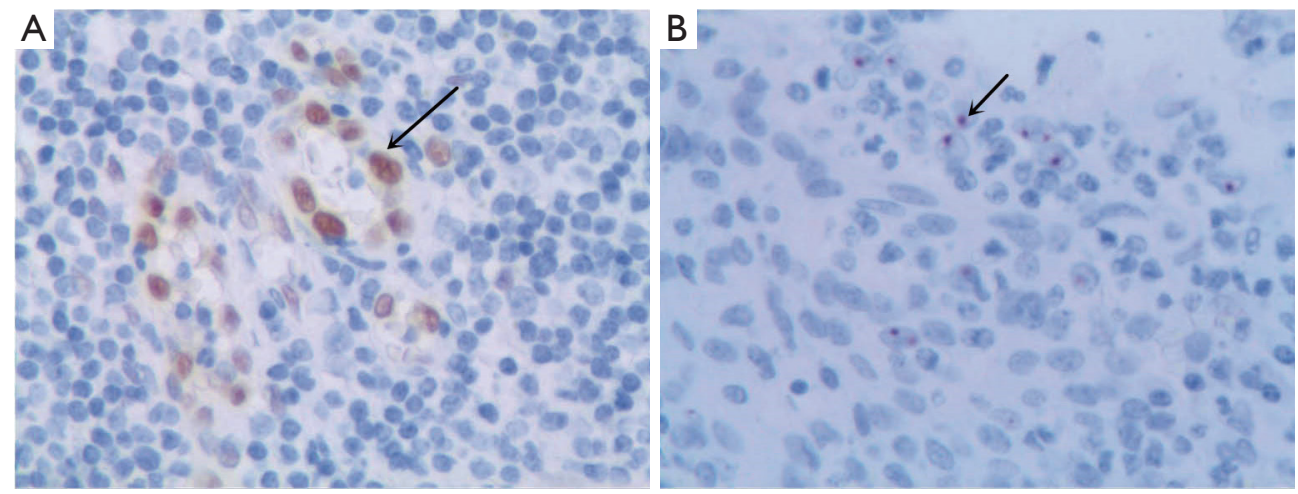

Figure 2 Immunostaining results for IL-33 (A) and ST2 (B) respectively. $\times 100$. The black arrow in (A) indicates IL-33 positive cells, the black arrow in (B) indicates ST2 positive cells. IL-33, interleukin-33; ST2, suppressor of tumorigenicity 2.
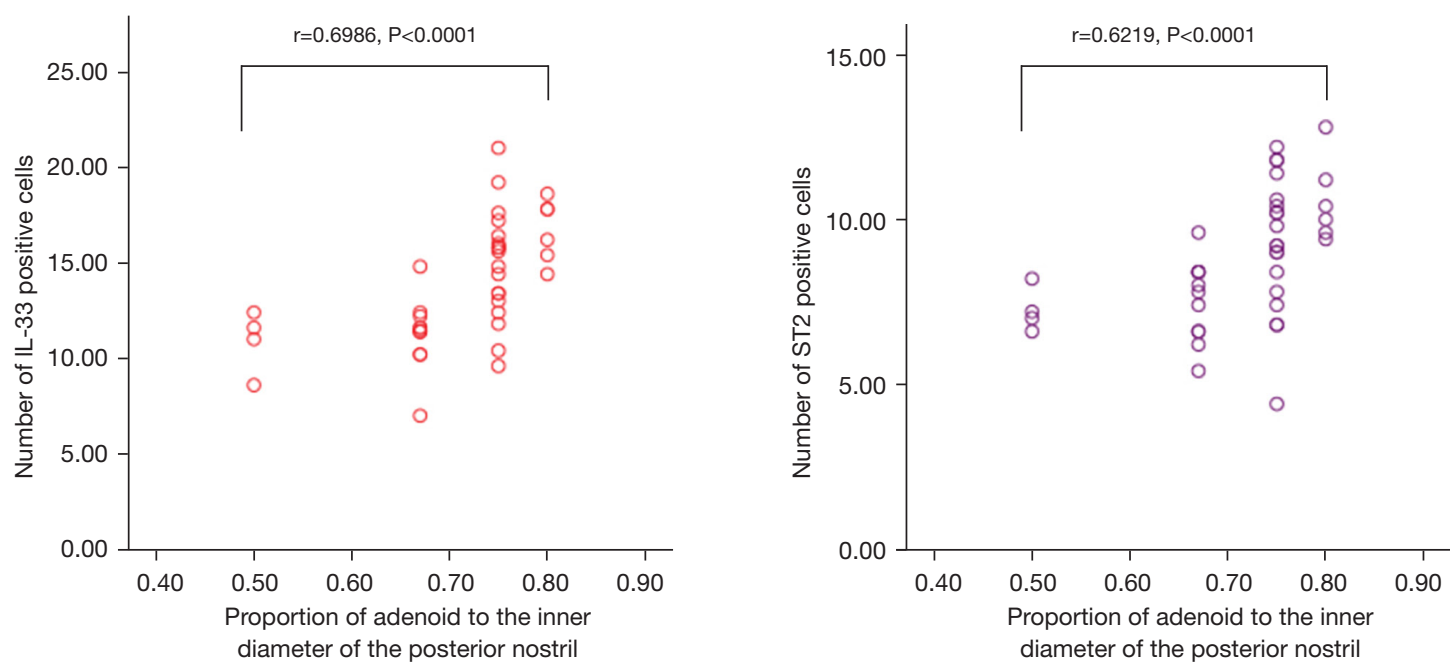

Figure 3 The correlations between the number of IL-33, ST2 positive cells and the proportion of adenoid to the inner diameter of the posterior nostril measuring by Spearman's correlation analysis with Prism 6 software. IL-33, interleukin-33; ST2, suppressor of tumorigenicity 2 .

the number of eosinophil granulocytes in adenoids $(\mathrm{r}=0.814$, $\mathrm{P}=0.000$ ), shown in Figure 4 .

\section{Discussion}

Adenoidal hypertrophy is a multifactorial, long-term process that could be broadly divided into infectious and non-infectious causes, including a variety of pathogens, gastroesophageal reflux, allergens or nasal secretions stimulation, and so on, which leads to adenoid proliferation and fibrosis (17-20). Tumor necrosis factors, inflammatory cytokines (IL-2, IL-4, IL-6), lipid peroxidation, and cell-free
DNA have been found to increase in OSAS patients (21). The expression of IL-33 occurs in various mucosal systems, which are the elicitation sites of immune response as well as the front line of defense against the external environment. Under normal physiological conditions, IL-33 protein is present in the nucleus of cells (22). When the corresponding tissues are infected or stimulated, IL-33 is released from the nucleus of the cells of the injured tissues to the nucleus (23).

The cytokine IL-33 binds to ST2, and is followed by the activation of ILC2, which can induce many inflammatory mediators such as IL- 6 , tumor necrosis factor- $\alpha$ (TNF- $\alpha$ ), IL-5, and IL-13, and promote Th0 to Th2 (24). The IL- 

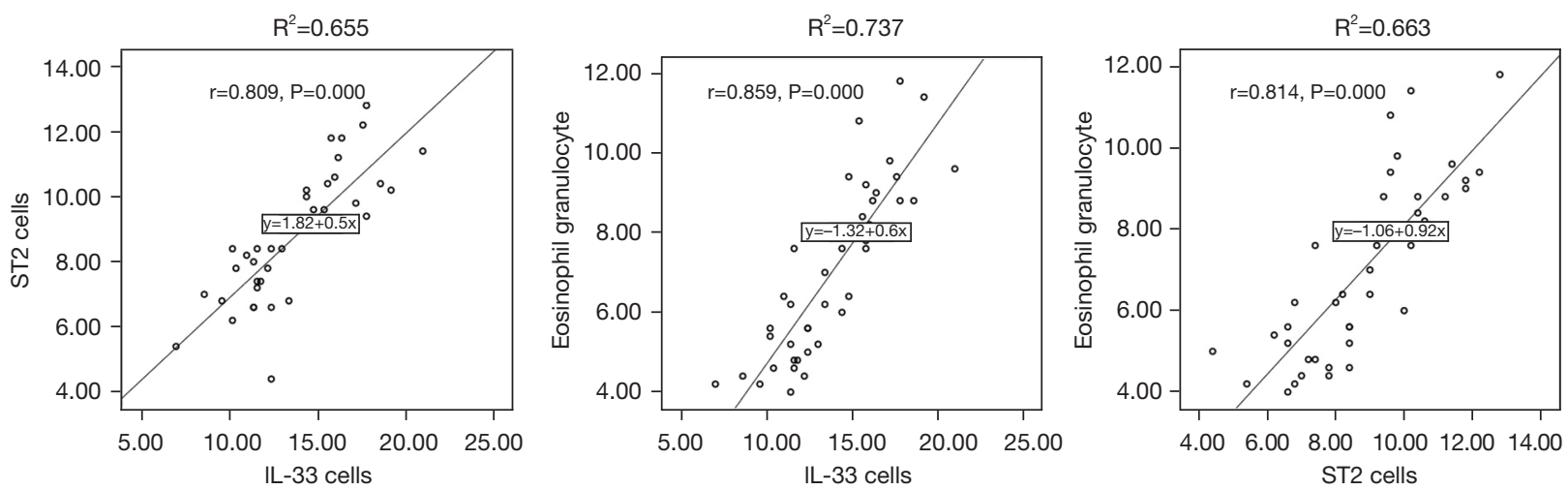

Figure 4 The correlations between the expression of IL-33, ST2 and the amount of eosinophil granulocytes in adenoids. IL-33, interleukin-33; ST2, suppressor of tumorigenicity 2.

33/ST2 pathway may be involved in the pathogenesis of a variety of human diseases, including autoimmune diseases such as systemic lupus erythematosus and dermatomyositis, allergic diseases such as asthma and idiopathic dermatitis (16), and even serious infectious diseases (24). It has been suggested that the IL-33/ST2 pathway and ILC2 may be involved in the inflammation, repair, and hypertrophy of adenoids. In this study, the expression of IL-33/ST2 in the adenoids of children with OSAS was confirmed, and the expression level of IL-33 was significantly different among different adenoids $(\mathrm{P}<0.01)$. The Spearman correlation coefficient was 0.6986 , $\mathrm{P}<0.0001$, suggesting that there was a positive correlation between the level of IL-33 expression and the degree of adenoid hypertrophy. In addition, the expression of IL-33 and its receptor ST2 was positively correlated with the level of eosinophil granulocyte count in adenoids. At the same time, the IL-33/ST2 pathway in adenoids could potentially be involved in the process of adenoid hypertrophy through up-regulation of eosinophil granulocytes.

\section{Conclusions}

Both IL-33 and its receptor ST2 were expressed in the adenoids of children with OSAS, which may play a key role in the pathogenesis of OSAS. There was a positive correlation between the expression of IL-33 and ST2 in adenoids. The higher the expression of IL-33 and ST2 in adenoids, the more eosinophil granulocytes in adenoids, but there was no correlation with the number of peripheral blood eosinophil granulocytes. The severity of airway obstruction caused by adenoid hypertrophy was positively correlated with the expression of IL-33/ST2.

\section{Acknowledgments}

Funding: None.

\section{Footnote}

Reporting Checklist: The authors have completed the MDAR reporting checklist. Available at https://tp.amegroups.com/ article/view/10.21037/tp-21-606/rc

Data Sharing Statement: Available at https://tp.amegroups. com/article/view/10.21037/tp-21-606/dss

Conflicts of Interest: All authors have completed the ICMJE uniform disclosure form (available at https://tp.amegroups. com/article/view/10.21037/tp-21-606/coif). The authors have no conflicts of interest to declare.

Ethical Statement: The authors are accountable for all aspects of the work in ensuring that questions related to the accuracy or integrity of any part of the work are appropriately investigated and resolved. All procedures performed in this study involving human participants were in accordance with the Declaration of Helsinki (as revised in 2013). This study was approved by the Bioethics Committee at Tianjin Children's Hospital (2021-YKY-04). A written consent form for publication of data was provided by the parents of participants.

Open Access Statement: This is an Open Access article 
distributed in accordance with the Creative Commons Attribution-NonCommercial-NoDerivs 4.0 International License (CC BY-NC-ND 4.0), which permits the noncommercial replication and distribution of the article with the strict proviso that no changes or edits are made and the original work is properly cited (including links to both the formal publication through the relevant DOI and the license). See: https://creativecommons.org/licenses/by-nc-nd/4.0/.

\section{References}

1. Schechter MS; Section on Pediatric Pulmonology, Subcommittee on Obstructive Sleep Apnea Syndrome. Technical report: diagnosis and management of childhood obstructive sleep apnea syndrome. Pediatrics 2002;109:e69.

2. Gozal D, Kheirandish-Gozal L. Obesity and excessive daytime sleepiness in prepubertal children with obstructive sleep apnea. Pediatrics 2009;123:13-8.

3. American Academy of Sleep Medicine. International classification of sleep disorders. USA: American Academy of Sleep Medicine: Darien, IL; 2014. Available online: https:// link.springer.com/chapter/10.1007/978-1-4939-6578-6_27

4. Marcus CL, Brooks LJ, Draper KA, et al. Diagnosis and management of childhood obstructive sleep apnea syndrome. Pediatrics 2012;130:e714-55.

5. Tan HL, Gozal D, Kheirandish-Gozal L. Obstructive sleep apnea in children: a critical update. Nat Sci Sleep 2013;5:109-23.

6. Brockbank JC. Update on pathophysiology and treatment of childhood obstructive sleep apnea syndrome. Paediatr Respir Rev 2017;24:21-3.

7. Huang YS, Guilleminault C. Pediatric Obstructive Sleep Apnea: Where Do We Stand? Adv Otorhinolaryngol 2017;80:136-44.

8. Gulotta G, Iannella G, Vicini C, et al. Risk Factors for Obstructive Sleep Apnea Syndrome in Children: State of the Art. Int J Environ Res Public Health 2019;16:3235.

9. Van de Graaff WB. Thoracic traction on the trachea: mechanisms and magnitude. J Appl Physiol (1985) 1991;70:1328-36.

10. Arens R, Sin S, Nandalike K, et al. Upper airway structure and body fat composition in obese children with obstructive sleep apnea syndrome. Am J Respir Crit Care Med 2011;183:782-7.

11. Kotsiou OS, Gourgoulianis KI, Zarogiannis SG. IL-33/ ST2 Axis in Organ Fibrosis. Front Immunol 2018;9:2432.
12. Cohen M, Hamilton J, Narang I. Clinically important agerelated differences in sleep related disordered breathing in infants and children with Prader-Willi Syndrome. PLoS One 2014;9:e101012.

13. Ruehland WR, Rochford PD, O'Donoghue FJ, et al. The new AASM criteria for scoring hypopneas: impact on the apnea hypopnea index. Sleep 2009;32:150-7.

14. Katz ES, D'Ambrosio CM. Pathophysiology of pediatric obstructive sleep apnea. Proc Am Thorac Soc 2008;5:253-62.

15. Kaditis AG, Alonso Alvarez ML, Boudewyns A, et al. Obstructive sleep disordered breathing in 2- to 18-yearold children: diagnosis and management. Eur Respir J 2016;47:69-94.

16. Drake LY, Kita H. IL-33: biological properties, functions, and roles in airway disease. Immunol Rev 2017;278:173-84.

17. Proenca-Modena JL, Paula FE, Buzatto GP, et al. Hypertrophic adenoid is a major infection site of human bocavirus 1. J Clin Microbiol 2014;52:3030-7.

18. Kim JH, Jeong HS, Kim KM, et al. Extra-Esophageal Pepsin from Stomach Refluxate Promoted Tonsil Hypertrophy. PLoS One 2016;11:e0152336.

19. Evcimik MF, Dogru M, Cirik AA, et al. Adenoid hypertrophy in children with allergic disease and influential factors. Int J Pediatr Otorhinolaryngol 2015;79:694-7.

20. Holm K, Bank S, Nielsen H, et al. The role of Fusobacterium necrophorum in pharyngotonsillitis - A review. Anaerobe 2016;42:89-97.

21. Maniaci A, Iannella G, Cocuzza S, et al. Oxidative Stress and Inflammation Biomarker Expression in Obstructive Sleep Apnea Patients. J Clin Med 2021;10:277.

22. Carriere V, Roussel L, Ortega N, et al. IL-33, the IL1-like cytokine ligand for ST2 receptor, is a chromatinassociated nuclear factor in vivo. Proc Natl Acad Sci U S A 2007;104:282-7.

23. Cayrol C, Girard JP. IL-33: an alarmin cytokine with crucial roles in innate immunity, inflammation and allergy. Curr Opin Immunol 2014;31:31-7.

24. Xu H, Turnquist HR, Hoffman R, et al. Role of the IL-33ST2 axis in sepsis. Mil Med Res 2017;4:3.

Cite this article as: Zhang Z, Li L, Zhao L, Liu G, Han F, $\mathrm{Du}$ J, Liu L. Expression and clinical significance of IL-33 and its receptor ST2 in children with obstructive sleep apnea syndrome. Transl Pediatr 2022;11(1):108-113. doi: 10.21037/ tp-21-606 